(MINI REVIEW ARTICLE)

\title{
Correlation between low platelets and malaria disease - Mini review
}

\author{
Omer Ibrahim Abdallah Mohammed *, Eihab Abdin Abdelaziz Gadelmula and Abdelmunim Izzeldin \\ Abderahman Dafalla
}

Sharjah Municipality, Department of Public Health Clinic, Sharjah- United Arab Emirates.

Publication history: Received on 01 September 2020; revised on 07 September 2020; accepted on 09 September 2020

Article DOI: https://doi.org/10.30574/wjarr.2020.7.3.0339

\begin{abstract}
Malaria is one of the major causes of disease for people living in tropical and subtropical areas. Infection with malaria parasites may result in a wide variety of symptoms, ranging from absent or very mild symptoms to severe disease and even death. Low platelets are common in the laboratory feature among patients with malaria. Causes of Low platelets in malaria till now it not clarified. In this mini review we try to find the correlation between malaria and thrombocytopenia. Malaria is associated with different degrees of low platelet count and the relationship between low platelets and malaria till now not sufficiently understood till now.
\end{abstract}

Keywords: Low Platelets; Thrombocytopenia; Correlation; Plasmodium; Malaria Disease

\section{Introduction}

Malaria is one of the major causes of disease for people living in tropical and subtropical areas. Despite intensive control efforts during the twentieth century, approximately $40 \%$ of the world's population remains at risk of infection. Malaria disease is parasitic infection caused by plasmodium species specially falciparum \& vivax, malariae, and ovale [1]. Thrombocytopenia is common in the laboratory diagnosis of malaria and presence of thrombocytopenia is not a distinguishing feature between the two types of malaria. Its presence in patients who present with acute febrile illness in the tropics, increases the probability of malaria [2]. Falciparum malaria associated with several hematological changes that affect the major blood cell lines such as platelets lead to platelets parameters (platelets count and indices) abnormalities[3]. Hematological changes are some of the most common complications in malaria and they play a major role in malaria pathogenesis. Patients infected with malaria exhibited important changes in most of hematological parameters with low platelet, WBCs, and lymphocyte counts being the most important predictors of malaria infection [4]. Alterations of blood coagulation are thought to be involved in malaria pathogenesis. Also, in malaria patients without clinically evident bleeding or thrombotic complications, alterations of the blood coagulation system, such as decreased levels of plasma antithrombin or elevated levels of plasminogen activator inhibitor (PAI)-1, and thrombocytopenia are frequently found $[1,5,6]$.

Most of researchers reported that, Anemia thrombocytopenia are the most frequent malaria-associated hematological complications and have received more attention in the scientific literature due to their associated mortality $[7,8]$. The anemia is usually due to varied reasons ranging from hemolysis to comorbidities like parasitic infections, folate, iron, and vitamin B12 deficiencies in endemic areas, antimalarials and further complicated by the coexistence of thalassemia and other haemoglobinopathies $[9,10]$. Regarding to thrombocytopenia during malaria however is yet to be understood clearly and what the mechanism exactly leads to lower the number of thrombocytes during malaria, some researchers hypothesize that malaria parasite produces factors that reduce the platelet production from the megakaryocytes. This saves the parasite from the platelet mediated clearance as a survival mechanism [11]. Other studies reported the mechanisms leading to thrombocytopenia are coagulation disturbances, splenomegaly, bone marrow alterations,

* Corresponding author: Omer Ibrahim Abdallah Mohammed
Sharjah Municipality, Department of Public Health Clinic, Sharjah- United Arab Emirates.

Copyright $(2020$ Author(s) retain the copyright of this article. This article is published under the terms of the Creative Commons Attribution Liscense 4.0. 
antibody-mediated platelet destruction, oxidative stress, and the role of platelets as cofactors in triggering severe malaria $[9,12,13,14,15]$. Fajardo and Tallent demonstrated Plasmodium vivax within platelets by electron microscopy and suggested a direct lytic effect of the parasite on the platelets [16]. Multiple studies indicate that platelets appear to both ameliorate infection by killing parasites and compromise the host by mediating adhesion of infected erythrocytes to the vascular wall. Herein, the possible causes of malaria-induced thrombocytopenia as well as the roles of the platelets in the erythrocytic stage of the disease will be discussed [17]. Another study conducted by Steven Kho et al., demonstrates the direct pathogen-killing actions and host protective roles of platelets during human malarial infection. Given platelets show broad-spectrum antimicrobial activity, and the risks for infection associated with thrombocytopenia [18]. Another hypothesis released by R. Conte et al., that autoantibodies against platelet glycoproteins IIb-IIIa and Ia-IIa might be present during malaria and could lead to severe thrombocytopenia [19]. Lee $\mathrm{SH}$ et al., conducted clinical trial in plasma macrophage colony-stimulating factor and P-selectin levels in malaria and they suggest that elevated M-CSF in malaria, by enhancing macrophage activity, may result in increased macrophagemediated platelet destruction [20]. Another study conducted by Casals et al., in Kenya about thrombocytopenia among patients with falciparum malaria and find thrombocytopenia strongly correlates with high levels of interleukin (IL)-10 [21]. Kotepui et al., observed patients during infected with malaria exhibited important changes in most of hematological parameters with low platelet, WBCs, and lymphocyte counts being the most important predictors of malaria infection. When used in combination with other clinical and microscopy methods, these parameters could improve malaria diagnosis and treatment [22]. Another study conducted by Khalid et al. concluded that the thrombocytopenia associated commonly with severe falciparum malaria and falciparum malaria hyperparasitemia particularly severe thrombocytopenia (PLTs $>50 \times 109 / \mu$ l).So thrombocytopenia and their severity may help to assess the disease severity and to improve the management of falciparum malaria among patients [23]. Malaria is associated with different degrees of low platelet count generally mild or moderate and bleeding is rare even during severe malaria. The relationship between thrombocytopenia and malaria not sufficiently understood till now [24]. Also another cause for thrombocytopenia should be considered because there many normal and abnormal condition or environmental can lead to change in platelets numbers if it coming associate with malaria like, heart disease, hypertension and pregnancy thrombocytopenia (platelets $100 \times 109 / \mathrm{L}$ ) occurs in $0.8 \%-0.9 \%$ of normal pregnant women, while increases in platelet factor and thromboglobulin suggest elevated platelet activation and consumption $[25,26,27,28]$. Thrombocytopenia may not be a cause of mortality by itself, but it can be a marker of increased severity and need of aggressive management [29].

\section{Conclusion}

Malaria is associated with different degrees of low platelet count and the relationship between low platelets and malaria till now not sufficiently understood till now.

\section{Compliance with ethical standards}

\section{Disclosure of conflict of interest}

The authors have declared that no competing interests exist.

\section{References}

[1] Mohammed 0, Abdalla M. Determination of Prothrombin Time, Activated Partial Thromboplastin Time and DDimer Levels among Malaria Infected Patients in Sinnar State, Sudan. Open Access Library Journal. 2019; 6: 1-6.

[2] Gill MK, Makkar M, Bhat S, Kaur T, Jain K, Dhir G. Thrombocytopenia in malaria and its correlation with different types of malaria. Ann Trop Med Public Health. 2013; 6: 197-200.

[3] Mohamedahmed, Khalid Abdelsamea, et al. Impact of Sever Plasmodium falciparum infection on Platelets Parameters among Sudanese children Living in Al-Jazira State. International Journal of Clinical and Biomedical Research. 2020; 5-9.

[4] Angchaisuksiri P. Coagulopathy in malaria. Thromb Res. 2014; 133: 5-9.

[5] Riedl J, Mordmüller B, Koder S, Pabinger I, Kremsner PG, Hoffman SL, Ramharter M, Ay C. Alterations of blood coagulation in controlled human malaria infection. Malaria journal. 15, 15.

[6] Asaad Ma. Babker. The role of Inherited Blood Coagulation Disorders in Recurrent Miscarriage Syndrome. Journal of Critical Reviews. 2020; 7(1): 16-20. 
[7] Lacerda MVG, Mourão MPG, Coelho HCC, Santos JB. Thrombocytopenia in malaria: who cares?. Memorias do Instituto Oswaldo Cruz. 2011; 106, 52-63.

[8] Wickramasinghe SN, Abdalla SH. Blood and bone marrow changes in malaria. Baillieres Best Pract Res Clin Haematol. 2000; 13: 277-299.

[9] Khan SJ, Abbass Y, Marwat MA. Thrombocytopenia as an indicator of malaria in adult population. Malaria research and treatment. 2012.

[10] K Ghosh and K Ghosh. Pathogenesis of anemia in malaria: a concise review, Parasitology Research. 2007; 101(6): 1463-1469.

[11] Srivastava K, Sharma M, Mitchell WB. (2017). Malaria and Thrombopoiesis: A Possible Mechanism for the Malarial Thrombocytopenia. Of. 6, 2.

[12] A Rasheed, S Saeed, SA Khan. Platelet count in malaria, Pakistan Journal of Phytopathology. 2008; 19(3), 86-88.

[13] SS Jamal Khan, FR Khan, M Usman, S Zahid. Malaria can lead to thrombocytopenia, Rawal Medical Journal. 2008; 33(2): 183-185.

[14] TS Faseela, RA Roche, KB Anita, CS Malli, Y Rai. Diagnostic value of platelet count in malaria, Journal of Clinical and Diagnostic Research. 2011; 5(3): 464-466.

[15] Mustafa ME, Mansoor MM, Mohammed A, Babker AAA. Evaluation of platelets count and coagulation parameters among patients with liver disease. World Journal of Pharmaceutical Research. 2005; 4(10): 360-368.

[16] Fajardo LF, Tallent C. Malarial parasites within human platelets. JAMA. 1974; 229: 1205-9.

[17] McMorran BJ. (2019). Immune role of platelets in malaria. ISBT Science Series. 14(1), 67-76.

[18] Kho S, Barber BE, Johar E, Andries B, Poespoprodjo JR, Kenangalem E, Woodberry T. (2018). Platelets kill circulating parasites of all major Plasmodium species in human malaria. Blood, The Journal of the American Society of Hematology. 132(12), 1332-1344.

[19] Conte R, Tassi C, Belletti D, Ricci F, Tazzari PL. Autoimmune thrombocytopenia in malaria. Vox sanguinis. 2003; 85(3), 221-221.

[20] Lee SH, Looareesuwan S, Chan J, Wilairatana P, Vanijanonta S, Chong SM, Chong BH. Plasma macrophage colonystimulating factor and P-selectin levels in malaria-associated thrombocytopenia. Thrombosis and haemostasis. 1997; 77(2): 289-293.

[21] Casals-Pascual C, Kai O, Newton CR, Peshu N, Roberts DJ. Thrombocytopenia in falciparum malaria is associated with high concentrations of IL-10. The American journal of tropical medicine and hygiene. 2006; 75(3): $434-436$.

[22] Kotepui M, Phunphuech B, Phiwklam N, Chupeerach C, Duangmano S. Effect of malarial infection on haematological parameters in population near Thailand-Myanmar border. Malaria journal. 2014; $13: 218$.

[23] Mohamedahmed, Khalid Abdelsamea, et al. Diagnostic and prognostic value of thrombocytopenia severity in Sudanese children with Falciparum malaria. World Journal of Advanced Research and Reviews 6.3. (2020); 197204.

[24] Babker AM. Is thrombocytopenia considered a valuable indicator tool for malaria? GSC Advanced Research and Reviews. 2020; 2(3), 052-054.

[25] Agabeldour AA, Khalafallah TO, AbdAllah AM. Hematological changes among Sudanese petroleum workers with a broad range of benzene exposure: Sch. J. App. Med. Sci. 2015; 3(8D): 3054-3056.

[26] Babker AM, Di Elnaim EO. Hematological Changes During All trimesters in Normal Pregnancy. Journal of Drug Delivery and Therapeutics. 2020 Mar 15; 10(2): 1-4.

[27] Eldour, Ahmed Abdalla Agab, et al. Fibrinogen Levels in Hypertensive and Normotensive: A Cross-Sectional Study from El-Obied City, Sudan. Journal of Biosciences and Medicines 4.2. (2016); 28-32.

[28] Monwara K. Methylenetetrahydrofolate reductase c677t polymorphism in Sudanese women with recurrent spontaneous abortions. Kuwait Medical Journal. 2016; 48(2). 100-104.

[29] Arti Muley, Jitendra Lakhani, Saurabh Bhirud, Abhinam Patel. Thrombocytopenia in Plasmodium vivax Malaria: How Significant?, Journal of Tropical Medicine. 2014; 4. 